\title{
The Boguslawski Melting Model
}

\author{
Vladimir Kh. Kozlovskiy \\ Scientific Society, Berlin, Germany \\ Email: Kozlovskiy.V@t-online.de
}

Received 15 December 2015; accepted 21 February 2016; published 24 February 2016

Copyright (C) 2016 by author and Scientific Research Publishing Inc.

This work is licensed under the Creative Commons Attribution International License (CC BY).

http://creativecommons.org/licenses/by/4.0/

\begin{abstract}
The anharmonic vibrator, whose expression of potential energy contains second and third powers of coordinates, is treated on the basis of dynamical procedure, which presents the state of motion by means of mean position and mean amplitude of vibration. The divergent statistical integral comes here not into consideration. The free energy is represented through mean atomic displacement and developed in power series, retaining fourth degree. The graphs show that at certain temperature, the minimum in free energy disappears, and the atom escapes from the potential pit. A simple atomic model that represents this phenomenon is proposed and the influence of model dimension and pressure on melting temperature will be presented.
\end{abstract}

\section{Keywords}

Boguslawski Vibrator, Melting, Three Atomic Model, Atomic Interactions

\section{Introduction}

A simple atomic model, which describes the phenomenon of melting, was elaborated by Boguslawski [1] [2]. According to this model, the atom is exposed to the action of two forces-attraction force, directed to the equilibrium position, and repulsive force, directed away from it. The total potential energy of an atom as a function of abscissa $x$ of the atom with origin in the position of equilibrium (minimum of energy) is represented by the expression

$$
U=\stackrel{0}{U}+\frac{c}{2} x^{2}+\frac{a}{3} x^{3} ; c>0, a<0
$$

Here $\stackrel{0}{U}$ is a constant, which is absent in the original paper, and $c$, $a$ are elastic and anharmonic coefficients. Figure 1 is the graph of polynom (1).

At low temperature the atom vibrates in the region of minimum. When the temperature is high enough, the atom approaches the top of the energy curve and escapes from the potential pit, which signifies the beginning of melting phenomenon. For determining of melting temperature this author tries to calculate the statistical integral, 


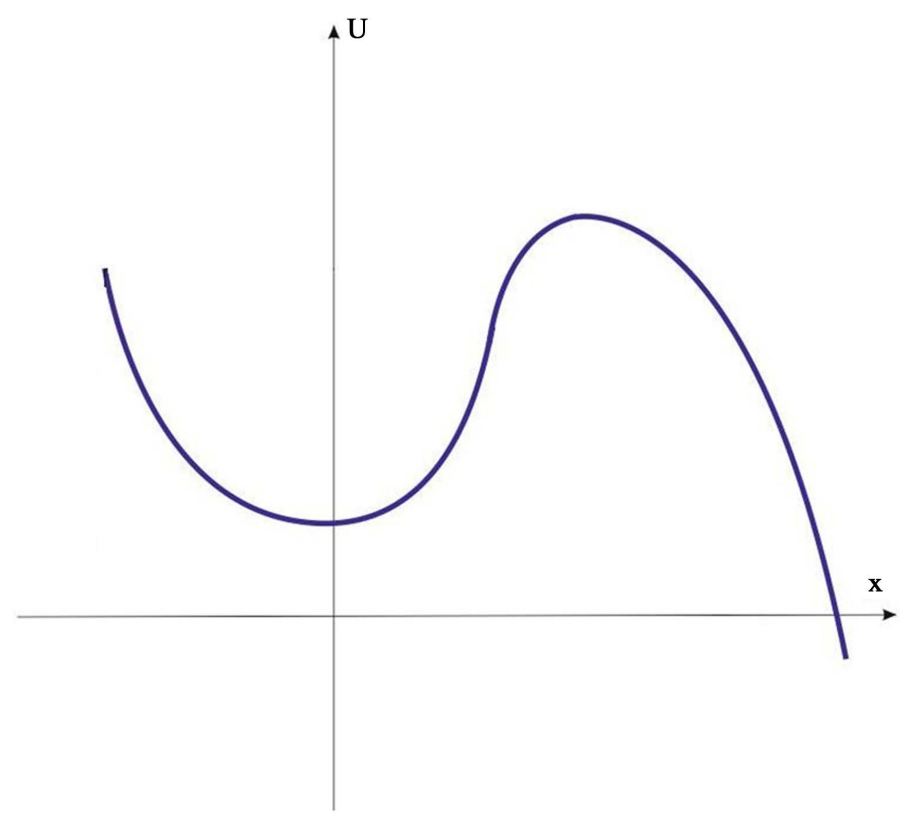

Figure 1. Potential energy of anharmonic vibrator.

but, unfortunately, it diverges in infinity for represented law of force. Boguslawski assumes that repulsive force is zero in space outside the top of potential curve, but attractive force increases as second power of abscissa, then the calculations is possible to carry out.

The behavior of potential pit outside the pit could have no meaning, if the atom was restricted in his movement to the pit only, but the statistics does not impose such a restriction.

The statistics permits the existents of a time moment, when the atom makes a jump over the potential barrier. In a regular crystal the movements of atoms are ordered and form in simple case a plane wave, so are mutually determined, and the determination lies in the laws of motion.

Braunbeck [3] solved the dynamical equation of motion of an atom in a potential field, described by sinusoidal function of coordinate, which represents the potential field in the crystal lattice. He established two types of motion - in the pit at low temperatures and above the top at high, which are in relation with solid and liquid states. So the melting phenomenon was demonstrated, but mathematical difficulties are considerable. It is worthwhile to use the form of dynamical equations that is able to calculate the mean thermal displacement of an atom and mean amplitude of vibration without solving time-dependent dynamical equations [4] [5]. The problem contains in solving algebraic equations, which may be carried out in some cases.

It is not excluded that the atoms in the lattice are fable bounded and behave almost independently. Then they can jump one by one over the potential barrier, which is an occasionally event, and the theory of transition must be developed in other direction.

Briefly, the described way of calculation is the following. Abscissa $x$ is represented in the form

$$
x=s+u \theta(t)
$$

where $s$ is the mean value of $x$ and $u$ is the mean value of amplitude of vibration, which is determined by equation

$$
u^{2}=\overline{(x-s)^{2}}
$$

The almost periodical function of time $\theta(t)$ satisfies the conditions $\bar{\theta}=0$ and $\overline{\theta^{2}}=1$. It will be assumed that $\overline{\theta^{3}}=0$, because the vibration function may be represented as a sum of trigonometric functions with incommensurable frequencies. Further, mean value of potential energy $\bar{U}$ may be calculated, and the variation principle for variables $s, u$ leads to the equations of state, where the temperature is a designation for mean kinetics energy 


$$
\frac{\partial \bar{U}}{\partial s}=0, u^{2} \frac{\partial \bar{U}}{\partial u^{2}}=\frac{1}{2} k T
$$

The expression for free energy $F$ has the form

$$
F=\bar{U}-\frac{k T}{2} \ln u^{2}
$$

The terms that depend on temperature only, are omitted, because they are not represented in the condition of minimum.

Boguslawski used his model of vibrator also for construction of the theory of pyroelectricity, and this work was mentioned in the article [6]. But for consideration of melting, it is useful to apply the way, elaborated by Ginsburg for considering Segnettelectricity [7] [8]. According to this way one must express the free energy through polarization $P$, which is proportional to $s$, develop in power series, and construct graphs $F(s)$ for different temperatures, which represent obviously the picture of phase transition.

\section{The Picture of Melting Phenomenon}

If the variable, that describes the state, is volume, then the conditions for melting are formulated in the work [9]. Volum and atom displacement (or polarizations) are similar, because they happen from place changing of atoms. As is shown in [9], the energy, arises from the change of volume, may be represented like for Boguslawski vibrator.

The free energy of Boguslawski vibrator is presented through expression

$$
F=\stackrel{0}{U}+\frac{c}{2}\left(s^{2}+u^{2}\right)+\frac{a}{3}\left(s^{2}+3 s u^{2}\right)-\frac{k T}{2} \ln u^{2}
$$

One of two equations of thermal equilibrium is written as

$$
\frac{\partial F}{\partial u^{2}}=\frac{c}{2}+a s-\frac{k T}{2 u^{2}}=0
$$

So, the amplitude is

$$
u^{2}=\frac{k T}{c+2 a s}
$$

Substituting in (4), we obtain after some calculation

$$
F=U_{0}-\frac{k T}{2} \ln \frac{k T}{e}+\frac{c}{2} s^{2}+\frac{a}{3} s^{3}+\frac{k T}{2} \ln (c+2 a s)
$$

Differentiating with respect to $s$ and equating the derivative to zero, we receive the second equation of thermal equilibrium and a connection between temperature and displacement

$$
\frac{\partial F}{\partial s}=c s+a s^{2}+\frac{a k T}{c+2 a s}=0
$$

So

$$
-a k T=s(c+a s)(c+2 a s)=2 a^{2} s^{3}+3 c a s^{2}+c^{2} s
$$

which gives an expression of temperature through displacement and with (6) also temperature through amplitude. The dependence $T(s)$ is represented on Figure 2, where physical meaning has the part of the graph from origin to maximum.

The dependence $T\left(u^{2}\right)$ of the form

$$
(k T)^{2}=c^{2}\left(u^{2}\right)^{2}-4 a^{2}\left(u^{2}\right)^{3}
$$

is represented through the curve with one maximum, which characterize the limiting values of variables. Further increase of the temperature leads to cease of the existence of state and to escape of the atom from the pit. Following expressions for coordinates at escaping are obtained 


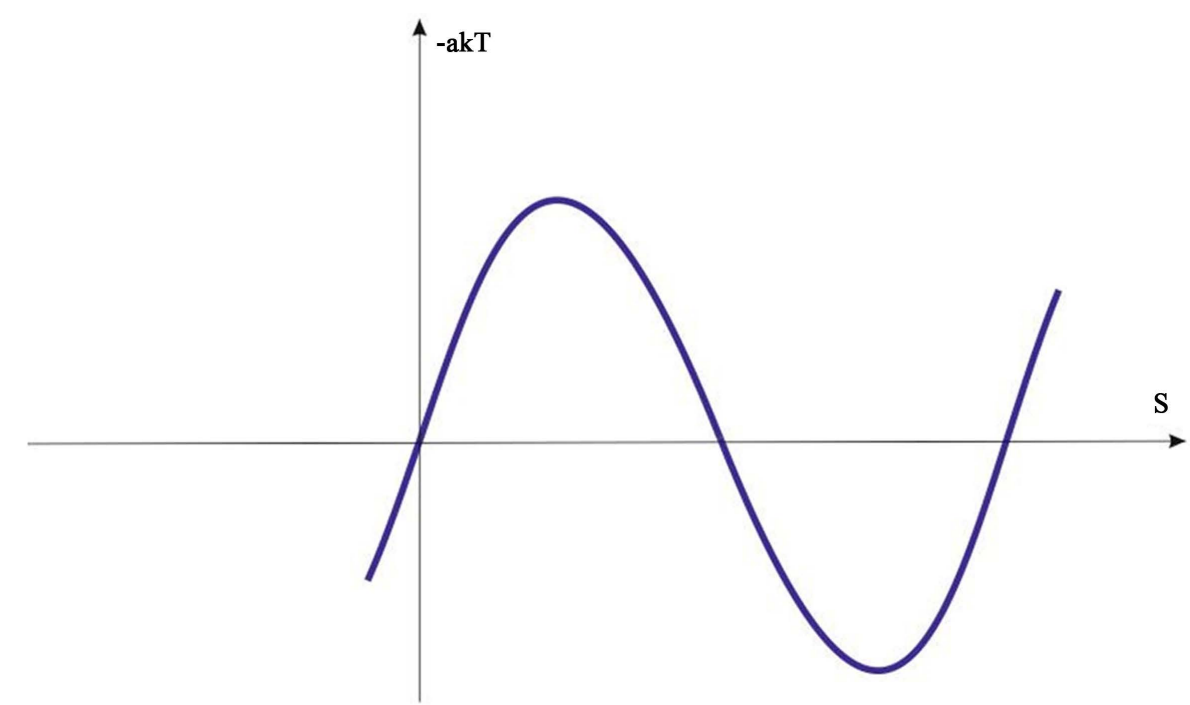

Figure 2. Dependence of temperature from displacement.

$$
s_{m}=-\frac{\sqrt{3}-1}{2 \sqrt{3}} \frac{c}{a} ; k T_{m}=\frac{c^{3}}{6 \sqrt{3} a^{2}} ; u_{m}^{2}=\frac{c^{2}}{6 a^{2}}
$$

Here the index $m$ marks the maximum of the curve.

So, the relation of amplitude to the displacement at melting point does not depend from the parameters of vibrator. Also, the coefficient of thermal elongation multiplied with temperature of melting does not contain parameters of vibrator. On may receive

$$
\frac{u_{m}}{s_{m}}=\frac{\sqrt{2}}{\sqrt{3}-1} ; \frac{1}{s_{m}}\left(\frac{\mathrm{d} s}{\mathrm{~d} T}\right)_{T=0} T_{m}=\frac{1}{3(\sqrt{3}-1)}
$$

These formulas can be compared with Grüneisen assertions [10] [11]. It may be remarked, that $s_{m}$ is a thermal elongation of interatomic distance, but not an interatomic distance itself, which cannot be calculated from the model. Because of acting of anharmonic forces, which diminish the restoring force, the quantity $c u_{m}^{2}$ is greater, then $k T_{m}$, namely $c u_{m}^{2}=\sqrt{3} k T_{m}$.

The temperature of minimum on Figure 2 has negative sign, but the same magnitude, as in expression (10).

Definite value of amplitude at melting point in relation to interatomic distance was offered by Lindemann as condition of melting [12], and this point of view was elaborated in the work of Gilvarry [13]. For Boguslawski vibrator the parameters of movement are obtained from the equations of state.

Further, the expansion will be used

$$
\ln (c+2 a s)=\ln c+\frac{2 a s}{c}-\frac{2 a^{2} s^{2}}{c^{2}}+\frac{8 a^{3} s^{3}}{3 c^{3}}+\cdots
$$

The expression for free energy takes the form of an expansion

$$
F=U_{0}-\frac{k T}{2} \ln \frac{k T}{e c}+\frac{a k T}{c} s+\frac{c}{2}\left(1-\frac{2 a^{2} k T}{c^{3}}\right) s^{2}+\frac{a}{3}\left(1+\frac{4 a^{2} k T}{c^{3}}\right) s^{3}+\cdots
$$

We may think that the first term includes the second term and under first term understand both. Graphs for low and high temperatures are represented on Figure 3 and Figure 4.

The abscissa of minimum (thermal equilibrium) is determined through the equation

$$
\frac{\partial F}{\partial s}=\frac{a}{c} k T+c\left(1-\frac{2 a^{2}}{c^{3}} k T\right) s+a\left(1+\frac{4 a^{2}}{c^{3}} k T\right) s^{2}=0
$$




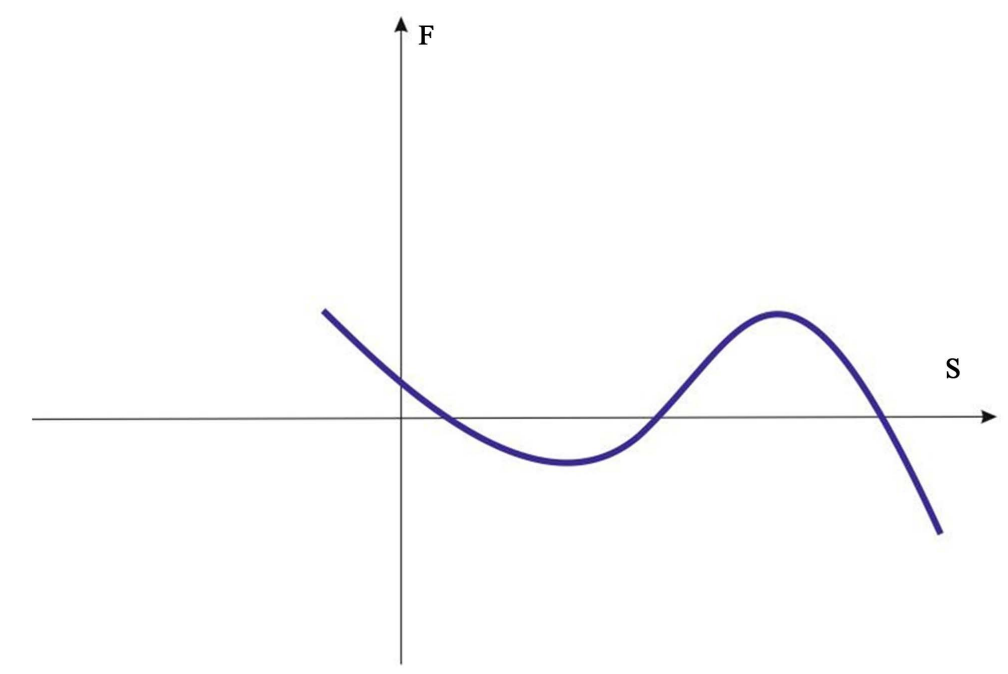

Figure 3. $F(s)$ at low temperature.

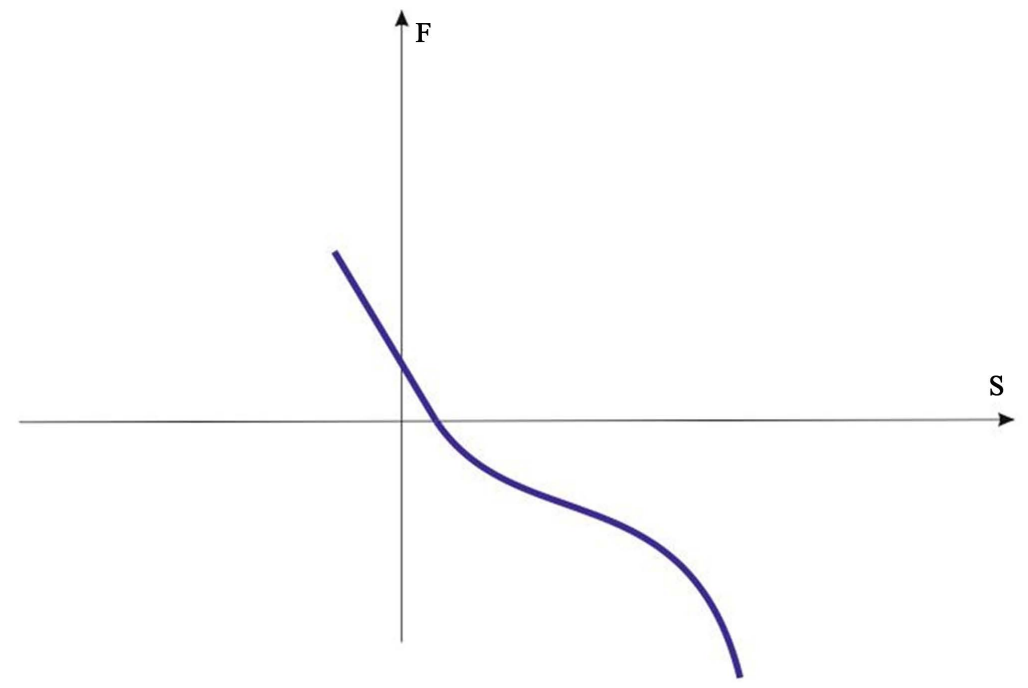

Figure 4. $F(s)$ at high temperature.

The temperature can be expressed as function of displacement and represented as power series.

By neglecting higher then second power of displacement this equation coincides with (9), so is valid for that part of Figure 2, which is placed between origin and maximum.

For low temperature one may put

$$
s=(s)_{k T=0}+\left(\frac{\partial s}{\partial k T}\right)_{k T=0} k T
$$

The first term is zero and the second is obtained through differentiating of (14). So, coordinates of minimum are given by means of expressions, where are retained terms quadratic in temperature

$$
s=-\frac{a}{c^{2}} k T ; \quad F=\stackrel{0}{U}-\frac{a^{2}}{2 c^{3}}(k T)^{2}
$$

When the temperature rises, the linear and cubic terms in (13) decreases as negative, the quadratic term decreases as positive, and the curve on the Figure 3 strives to be smooth. When both extremes coincide in a bending point with horizontal tangent, the corresponding temperature will be determined by means of (14) and con- 
dition

$$
\frac{\partial^{2} F}{\partial s^{2}}=c\left(1-\frac{2 a^{2}}{c^{3}} k T\right)+2 a\left(1+\frac{4 a^{2}}{c^{3}} k T\right) s=0
$$

Excluding from (14) and (17) the displacement, we receive the equation for the temperature

$$
\left(1-\frac{2 a^{2}}{c^{3}} k T\right)^{2}=\frac{4 a^{2}}{c^{3}} k T\left(1+\frac{4 a^{2}}{c^{3}} k T\right)
$$

Putting

$$
\frac{4 a^{2}}{c^{3}} k T=\varsigma
$$

we obtain an equation

$$
(2-\varsigma)^{2}=4 \varsigma(1+\varsigma)
$$

So $\zeta=0.43$, and temperature and displacement have in the bend point $B$ value

$$
k T_{B}=0.11 \frac{c^{3}}{a^{2}} ; s_{B}=-0.55 \frac{c}{2 a}
$$

which are not far from the values (10). The free energy at this point has the value

$$
F=\stackrel{0}{U}-0.0066 \frac{c^{3}}{a^{2}}
$$

These values may be regarded as parameters of melting. The corresponding graph is shown on Figure 5.

Here this phenomenon of melting is understood as disappearance of stable crystalline state without consideration of the liquid, but liquid state is also state with own properties, so that can hinder the movement of atom. The atom will vibrate during some time about equilibrium position, so that the model of anharmonic vibrator can be used for liquid state. Then the expression (16) for free energy with other values of coefficients (denoted by asterisk) may be an approximation to the free energy of liquid state

$$
F_{*}=\stackrel{0}{U_{*}}-\frac{a_{*}^{2}}{2 c_{*}^{3}}(k T)^{2}
$$

The condition of phase equilibrium consists in equality of expressions for free energies of both states

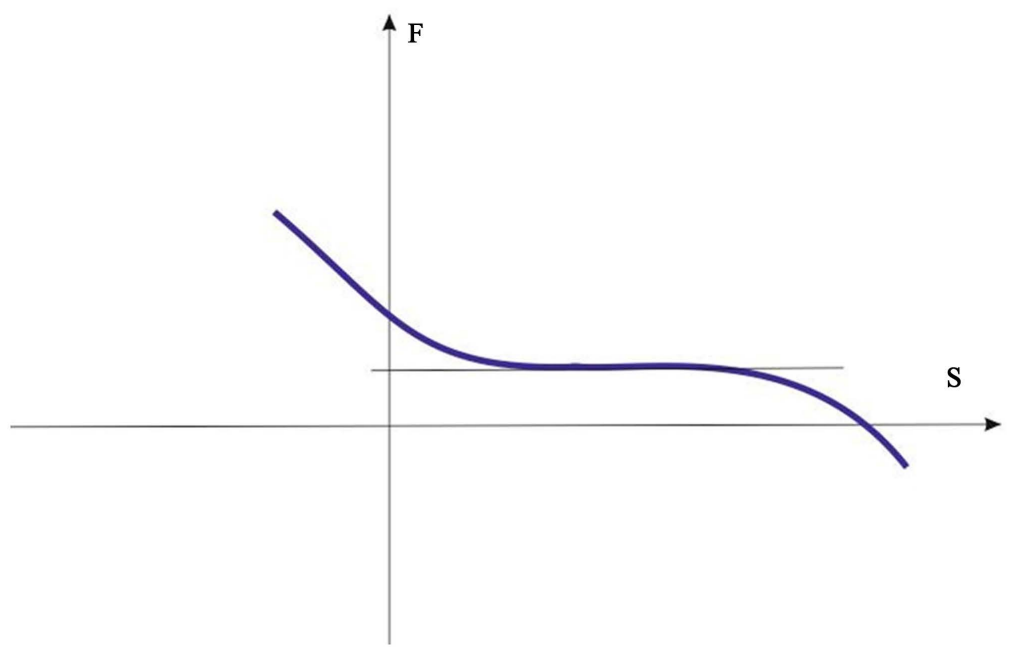

Figure 5. $F(s)$ for transition temperature. 


$$
\stackrel{0}{U}-\frac{a^{2}}{2 c^{3}}\left(k T_{e}\right)^{2}=\stackrel{0}{U_{*}}-\frac{a_{*}^{2}}{2 c_{*}^{3}}\left(k T_{e}\right)^{2}
$$

The temperature of equilibrium is equal

$$
\left(k T_{e}\right)^{2}=\frac{1}{3} \frac{\stackrel{0}{U}-\stackrel{0}{U_{*}}}{\frac{a^{2}}{6 c^{3}}-\frac{a_{*}^{2}}{6 c_{*}^{3}}}
$$

Nominator and denominator must be either positive or both negative. The denominator contains differences of barriers heights reciprocal values. The second case is probable, because in solid phase the depth of minimum is more significant and the height of barrier greater than in the liquid one. When the vibrations are considered as harmonic, the expression like (24) was obtained in the work [14].

In the work of Born [15], devoted to the melting phenomenon, condition of melting is formulated as disappearance of shearing resistance thanks to the action of fluidity. But this opinion is not precise, because fluidity exists, when mechanical action is of long duration. By short duration (firing in the liquid) fluidity is not manifested. When duration of mechanical action is very long, the solid corps also reveals fluidity, such behavior show a glacier. The question about rigidity and fluidity was elaborated in the work of Frenkel [16].

Brilloin also considers the question of temperature influence on elastic properties of solids, and disappearance of elastically resistance at some temperature is according his opinion a melting phenomenon [17].

The theory of Grüneisen was précised in our work [5] and about melting may be formulated following assertions. In Figure 6 of the mentioned work a family of isotherms is drawn, that are curves with maximum. The height of maximum (value of maximal applied stretching force) diminishes with increasing temperature till such temperature is achieved, that the top of the curve reaches the abscissa axis. The slop of the tangent to isotherm in the point of intersection with abscissa axis increases to the value $\pi$, then elasticity disappears. Probably the melting arises? But such opinion is imprudent, it can be only supposed, that the state is now a non equilibrium one and cannot be described by formulated equations. The moving atoms form a new structure, which comes to thermal equilibrium with primary and after a time, which can be much greater, then the period of atomic vibration, the fluid will be developed. In this time rearrangements of atoms between different sites can be realized.

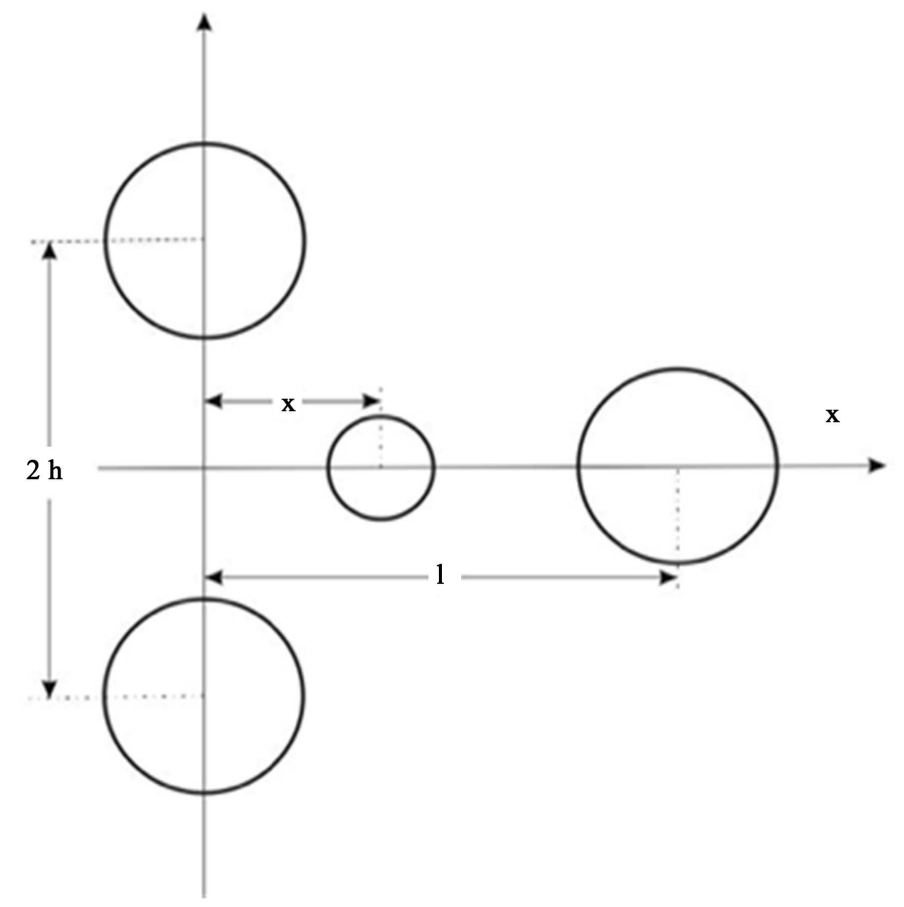

Figure 6. Four atomic model of vibrator. 


\section{Atomic Model of Anharmonic Vibrator}

The Boguslawski model is formal, because the parameters of the model are introduced as quantities determined from experiment. Later Frenkel [18] [19] developed a three atomic model of melting (three identical atoms placed on a straight line), where the middle atom interacts by means of atomic forces with others, that are immobile, and moves between them. It turns out, that in the middle point of interval between right and left atoms is placed a minimum of potential energy, if the atoms are near one to another, and maximum, if they are far. So, by changing of the length of the model phase transition may occur. But in this model the middle atom moves only between the others and cannot escape from the pit. It is worthwhile to replace the right or left atom through two identical atoms placed on ordinate from both sides of abscissa axis on equal distances, as is shown on Figure 6, where are marked the dimensions. Such model from other reasons was considered in the work [20]. Here is assumed, that the interactions, that are considered, are stipulated through the forces of repulsion, which is admissible, if the distances between atoms are small. The potential energy of the middle atom has the form

$$
U=\frac{2 \lambda}{\left[\sqrt{h^{2}+x^{2}}\right]^{n}}+\frac{\lambda}{(l-x)^{n}}
$$

It will be supposed, that $h<l$.

Further expansions will be used

$$
\begin{gathered}
\frac{1}{\left[\sqrt{h^{2}+x^{2}}\right]^{n}}=h^{-n}-\frac{n}{2} h^{-(n+2)} x^{2}+\cdots \\
\frac{1}{(l-x)^{n}}=l^{-n}+n l^{-(n+1)} x+\frac{1}{2} n(n+1) l^{-(n+2)} x^{2}+\frac{1}{6} n(n+1)(n+2) l^{-(n+3)} x^{3}+\cdots
\end{gathered}
$$

The potential energy of moving atom may be represented by the expression

$$
U=\frac{2 \lambda}{h^{n}}+\frac{\lambda}{l^{n}}+\frac{n \lambda}{l^{n+1}} x+\frac{1}{2}\left[-\frac{2 n \lambda}{h^{n+2}}+\frac{n(n+1) \lambda}{l^{n+2}}\right] x^{2}+\frac{n(n+1)(n+2) \lambda}{6 l^{n+3}} x^{3}+\cdots
$$

It will be assumed, that the action of right atom in the region of origin is small and may be omitted, so the linear term falls out and following expression may be accepted

$$
U=\frac{2 \lambda}{h^{n}}-\frac{1}{2} \frac{2 n \lambda}{h^{n+2}} x^{2}+\frac{1}{3} \frac{n(n+1)(n+2) \lambda}{2 l^{n+3}} x^{3}+\cdots
$$

The graph is represented on Figure 7.

The deduction is not rigorous, but physically we return to Boguslawski vibrator, where the ordinate axis goes through the maximum of potential energy. The elastic coefficient $c$ is now negative and $a$ positive, so the direction of temperature growing on Figure 2 is now reversed. Physical meaning has now the part of the graph, where displacement diminishes with rising of temperature, beginning from the value $c / a$ (minimum of energy). The general expression for transition temperature rests the same, as before. Using expression (20) for transition temperature, we receive

$$
k T_{B}=3.5 \frac{n \lambda}{(n+1)^{2}(n+2)^{2} l^{n}}\left(\frac{l}{h}\right)^{3 n+6}
$$

This formula is not suited for numerical calculations, because the ratio $l / h$ is presented in high power, so small variations of the fraction leads to great changes of temperature,. But it is possible to investigate the influence of length by constant ratio $l / h$ on transition temperature. In experiments the dimension is not considered as independent variable, but such is the stretching or, usually, compressing force. This influence can be calculated, using a simple model. When on two interacting with atomic forces atoms, separated through distance $l$ (molecule), are acting stretching forces $f$ and $-f$. the potential energy is

$$
U=\frac{\lambda}{l^{n}}-\frac{\mu}{l^{m}}-f l
$$




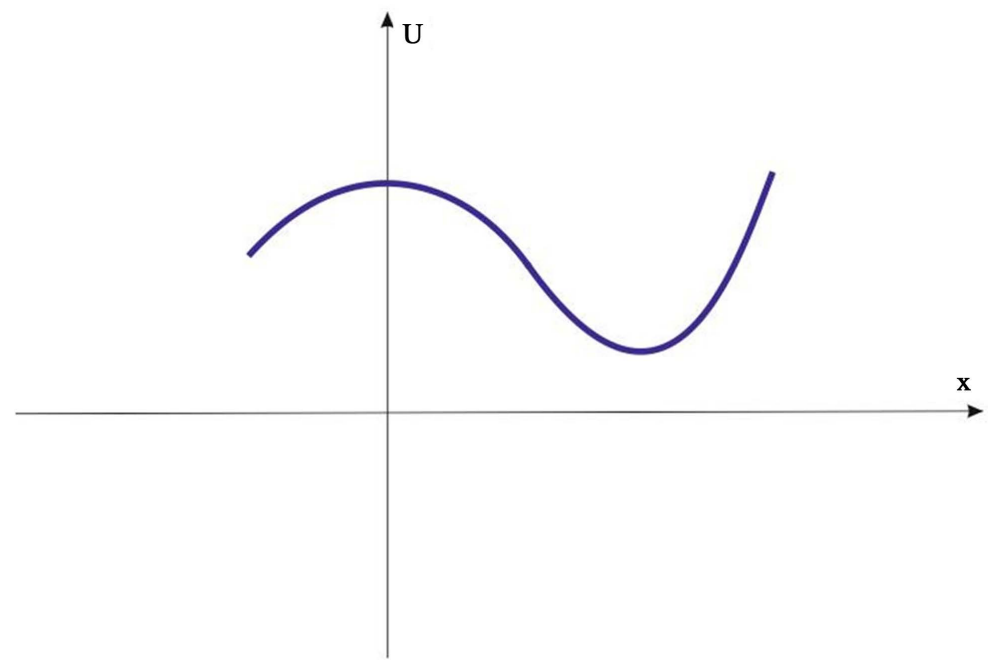

Figure 7. Potential curve in four atomic models.

The equilibrium distance in the absence of stretching forces is

$$
l_{0}=\left(\frac{n \lambda}{m \mu}\right)^{\frac{1}{n-m}}
$$

When the force has maximal value, corresponding to destruction of molecule (two extreme coincide in a bend point with horizontal tangent), the limiting distance has the value

$$
l_{i}=\left[\frac{n(n+1) \lambda}{m(m+1) \mu}\right]^{\frac{1}{n-m}}
$$

Corresponding limiting force is

$$
f_{i}=\frac{n(n-m)}{m+1} \frac{\lambda}{l_{i}^{n+1}}
$$

So, by increasing of stretching force from zero value to maximal one the distance between atoms increases from $l_{0}$ to $l_{i}$. Supposing linear dependence between distance and external force, we may put

$$
l=l_{0}+\frac{l_{i}-l_{0}}{f_{i}} f
$$

Approximately

$$
l^{-n}=l_{0}^{-n}-n \frac{l_{i}-l_{0}}{l_{0}^{n+1} f_{i}} f
$$

So, stretching leads to decrease and compressing to increase of the melting temperature according linear law. The latter phenomenon was observed in experiments [21]. More elaborate investigation shows, that connection between pressure and temperature for melting phenomenon is represented by means of non linear function [22].

In article [23] was pointed out, that the phenomenon of melting occurs, when the moving atom reaches the bend point of potential energy. The bend point in potential energy curve actually leads to peculiarities in behavior of the atom [20], but gives no possibility to escape from the potential pit.

The consideration of melting phenomenon with the help of a cubic cell model was undertaken in the work [24]. There the melting was treated as escaping from the cell, and as independent variable was regarded the length of the cell edge without introducing of the stretching force. This investigation will be continued.

So, the simple Boguslawski model of anharmonic vibrator can be used to describe in general features the phenomenon of melting on the base of dynamical principles. 


\section{Concluding Remarks}

Boguslawski in many of his works elaborated the theory of solid corps, based on the expression of potential energy of anharmonic vibrator with terms of second and third powers of coordinate. The statistical integral with such expression of energy diverges in infinity, and one cannot be sure that the way of calculation, employed by this author, leads to correct results. So, the received expression for the temperature of transition to the liquid state seems some uncertain. Calculations on the base of dynamical principles lead to utterly definite expression for the temperature of transition. This is so, because dynamical calculations treat the movement of atom in the pit as anharmonic vibrations with finite amplitude and without shift of atom in the infinity. But the calculation is not so simple, as in original paper. An atomic model, which creates a potential of Boguslawski vibrator is considered, and the parameters of Boguslawski expression of potential energy are presented in their dependence from parameters of atomic interaction, which include interatomic distances. So the influence of external pressure on the phenomenon of melting can be included in dynamical calculations.

In what relations to the experimental data are the results of calculation? After eliminating of infinite quantity, the Boguslawski formula for melting temperature could be compared with experimental data for some metals. It turned out that his formula differs from our only unimportant-the coefficient in denominator is marked $2 \eta^{2}$, where $\eta$ is determined from experimental data and approximately has for all metals the same value 1.89 , so the coefficient in denominator is 7.14 (in our formula 10.4). This means that a tenth of atoms escaped from regular

places when melting begins. Boguslawski gives also values for the ratio $c / a$, calculated from experimental data, so it is possible to calculate $S_{m}$ and compare with experimental determined interatomic distances, which are given in the table.

\section{References}

[1] Boguslawski, S.A. (1915) About the Mechanism of Melting Process. Transactions of Russian Physics-Chemical Society, Physics, 47, 540-545.

[2] Boguslawski, S.A. (1961) About the Mechanism of Melting Process. Selected Works in Physics, Edition with Notes of Prof. V.K. Sementchenko. State Edition for Physics-Mathematical Literature. Moscow, 99-104.

[3] Braunbeck, W. (1926) Grating Dynamical Theory of Melting Phenomenon. Physical Magazine, 38, 549-572.

[4] Kozlovskiy, V.Kh. (1991) Dynamical Equations for Time-Averaged Coordinates in Thermal Equilibrium. Soviet Physics Journal, 34, 353-359. http://dx.doi.org/10.1007/BF00898103

[5] Kozlovskiy, V.Kh. (2012) To the Question of Validity Grüneisen Solid State Equation. World Journal of Condensed Matter Physics, 2, 219-227. http://dx.doi.org/10.4236/wjcmp.2012.24038

[6] Born, M. and Brody, E. (1921) About the Vibration of Mechanical System with Finite Amplitude and Their Quantization. Physical Magazine, 6, 140-152.

[7] Ginsburg, V.L. (1945) About Dielectric Properties of Segnettelectrics and Barium Titanat. Journal of Experimental and Theoretical Physics, 15, 739-749.

[8] Ginsburg, V.L. (1949) The Theory of Segnettelectric Phenomena. Successes in Physical Sciences, 38, 490-525.

[9] Herzfeld, K.F. and Mayer, M.G. (1934) On the Theory of Fusion. Physical Review, 46, 995-1001. http://dx.doi.org/10.1103/PhysRev.46.995

[10] Grüneisen, E. (1911) To the Theory of One Atomic Solid Corps. Bulletin of German Physical Society, 13, .836-841.

[11] Grüneisen, E. (1912) Theory of One Atomic Elements Solid State. Annals of Physics, 39, 257-306. http://dx.doi.org/10.1002/andp.19123441202

[12] Lindemann, F.A. (1910) About the Calculation of Own Molecular Frequencies. Physical Papers, 11, $609-612$

[13] Gilvarry, J.J. (1956) The Lindemann and Grüneisen Laws. Physical Review, 102, 308-316. http://dx.doi.org/10.1103/PhysRev.102.308

[14] Kozlovskiy, V.Kh. (2003) Vibration-Diffusional Model of Melting. Transactions of VI International Conference of Crystals: Growth, Properties, Real Structure, Application, Alexandrov, 8-12 September 2003, 573-594.

[15] Born, M. (1939) Thermodynamics of Crystals and Melting. Journal of Chemical Physics, 7, 591-603. http://dx.doi.org/10.1063/1.1750497

[16] Frenkel, J.I. (1936) On the Liquid State and the Theory of Fusion. Physical Magazine, 47, 58-65.

[17] Brillouin, L. (1938) On Thermal Dependence of Elasticity in Solids. Physical Review, 54, 916-917. http://dx.doi.org/10.1103/PhysRev.54.916 
[18] Frenkel, J.I. (1935) The Liquid State and the Theory of Fusion. Physico-Chemical Works of URSS, 3, 632-648.

[19] Frenkel, J.I. (1958) The Liquid State and the Theory of Fusion. Collected Papers, 2, 269-279.

[20] Kozlovskiy, V.K. (2010) Model of Crystal Behavior in the Vicinity of the Bend Point of the Potential Curve for an Atom. Russian Physics Journal, 53, 1158-1166.

[21] Bridgeman, P.W. (1931) The Physics of High Pressure. Chap. VII, George Bell \& Sons, London.

[22] Simon, F.E. (1953) The Melting of Iron at high Pressure. Nature, 172, 746-747. http://dx.doi.org/10.1038/172746a0

[23] Rashevsky, N.V. (1926) To the Theorie of Melting Heats. Physical Magazine, 40, 214-219.

[24] Kozlovskiy, V.K. (1999) Dynamical Examination of Mechanical Behavior According to Three Atomic and Melting According to Nine Atomic Models of a Crystal. Transactions of IV International Conference Crystals: Growth, Properties, Real Structure, Application, 2, 466-476. 